\title{
Montmorillonite KSF and montmorillonite K-10 clays as efficient catalysts for the solventless synthesis of bismaleimides and bisphthalimides using microwave irradiation
}

\author{
Davood Habibi* and Omid Marvi \\ College of Chemistry, Bu-Ali Sina University, Hamedan, 6517838683, Iran \\ E-mail:dhabibi@basu.ac.ir
}

\begin{abstract}
Different bismaleimides and bisphthalimides were synthesized in a simple and environmentally benign method from the condensation reaction of maleic and phthalic anhydrides with different diamines on montmorillonite KSF and montmorillonite K-10 clays as solid acidic catalysts by microwave irradiations under the solvent-free conditions in good yields and short reaction times.
\end{abstract}

Keyword: Bismaleimides, bisphthalimides, montmorillonite K-10 and KSF clays, microwave

\section{Introduction}

Development of the simple and general synthetic routes for widely used organic compounds from the readily available reagents is one of the major challenges in organic synthesis. Maleimides are among such types of organic compounds which belong to an important class of substrates for chemical applications especially in biological, ${ }^{1-3}$ synthetic $^{4-6}$ and polymer chemistry. ${ }^{7,8}$ Maleimides react quickly with cysteine residues and thus are used as chemical probes of protein structure ${ }^{9}$ and as linkers for conjugation of molecules to proteins. ${ }^{10}$ These derivatives may in turn serve as immunoconjugates for cancer therapy, solid supported enzymes for synthetic applications, or haptens for production of antibodies. ${ }^{11}$ The maleimide functionality can be used as a synthetic platform in total synthesis due to its Michael-accepting ability and dienophilic nature. Also, thermally induced homopolymerized bismaleimides give rise to resins with good thermooxidative stability and low moisture absorption characteristics. ${ }^{12}$

Despite their wide applicability, available routes for the synthesis of these compounds are limited. ${ }^{13-16}$ On the other hand; each of these routes has its own synthetic problems when applied to a range of derivatives. For instance, synthesis of the functionalized maleimides either by direct condensation or through the intermediate acid cyclization method gives poor yields of desired maleimide derivatives.

Consequently, it is desirable to develop an easy manipulative procedure, as well as to avoid using the strong acids or bases and other corrosive media and replacing hazardous or 
expensive reactants and reagents by safer and economical ones. In achieving many of these goals, catalysts help the synthetic chemist in a big way. They are capable of making impracticable reactions to occur under the mildest possible conditions.

Montmorillonite clays have been used as catalysts for number of organic reactions and offer several advantages over classical acids. For example the strong acidity, non-corrosive properties, cheapness, mild reaction conditions, high yields and selectivity and the ease of setting and working-up. ${ }^{17}$

In the last few years a growing interest has been shown in the use of microwave irradiation in organic synthesis, also. ${ }^{18,19}$ In addition, microwave solvent-free synthesis offers advantages for reducing hazardous explosions and removal of the high boiling solvents from the reaction mixtures. $^{20}$

In continuation of our studies on microwave-assisted reactions on solid surfaces, ${ }^{21}$ herein we wish to describe a convenient and simple method for the synthesis of different bismaleimides and bisphthalimides from the condensation reaction between an appropriate mixture of maleic or phthalic anhydrides with different diamines on montmorillonite KSF and montmorillonite K-10 clays as solid acidic catalysts and microwave irradiations under the solvent-free conditions in good yields and short reaction times (scheme 1). A comparative data between our applied procedure and the conventional method has been shown in the general procedure section.

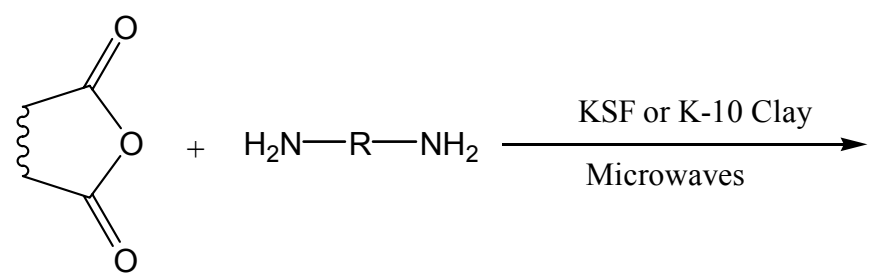

1) Maleic anhydride

2) Phthalic anhydride

3) 4-Chlorophthalic anhydride

4) 3,6-Dichlorophthalic anhydride

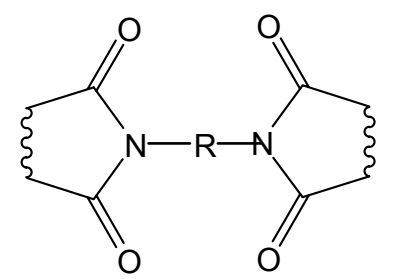

1a-h, 2a, 3a, 4a

\section{Scheme 1}

\section{Results and Discussion}

Several examples (22 entries) illustrating this simple and convenient method for the synthesis of bismaleimides and bisphthalimides were summarized in table 1. According to this table, the percentage of the products obtained by the KSF clay is more than those prepared by the K-10 clay, while the reaction times with the montmorillonite K-10 clay are less. This observation can be attributed to the more surface area in K-10 and the more acidic character of the KSF clay, 
respectively. ${ }^{22 \mathrm{~g}}$ These two characteristics usually compete with each other and due to both the small reaction time and the vigorous molecular vibrations in the microwave systems, the exact conclusion is not easy and needs further experiments, apparently.

Application of the aromatic diamines gives more yields rather than the aliphatic ones which might be related to the different stability of the products.

Table 1. The yields and reaction times for the synthesis of compounds $\mathbf{1 a - h}, \mathbf{2 a}, \mathbf{3 a}$ and $\mathbf{4 a}$ on montmorillonite KSF and K-10 clays

\begin{tabular}{|c|c|c|c|c|c|c|}
\hline No. & Diamine & Product* & $\begin{array}{c}\text { Crystallization } \\
\text { solvent }\end{array}$ & Clay & $\begin{array}{l}\text { Time } \\
(\min )\end{array}$ & $\begin{array}{c}\text { Yield** } \\
(\%)\end{array}$ \\
\hline 1 & & & & KSF & 7.5 & 86 \\
\hline 2 & & & Dioxane & K10 & 5.5 & 81 \\
\hline 3 & $\mathrm{H}_{2} \mathrm{~N}\left(\mathrm{CH}_{2}\right)_{2} \mathrm{NH}_{2}$ & & & KSF & 8 & 74 \\
\hline 4 & & 1b & $\mathrm{CH}_{2} \mathrm{Cl}_{2} /$ Ether & $\mathrm{K} 10$ & 6 & 69 \\
\hline 5 & & & & KSF & 8 & 68 \\
\hline 6 & $\mathrm{H}_{2} \mathrm{~N}\left(\mathrm{CH}_{2}\right)_{6} \mathrm{NH}_{2}$ & 1c & $\mathrm{CH}_{2} \mathrm{Cl}_{2} /$ Ether & K10 & 6.5 & 61 \\
\hline 7 & & & & KSF & 6 & 89 \\
\hline 8 & & & & K10 & 4.5 & 82 \\
\hline 9 & & & & KSF & 6.5 & 87 \\
\hline 10 & & & & K10 & 4.5 & 78 \\
\hline
\end{tabular}




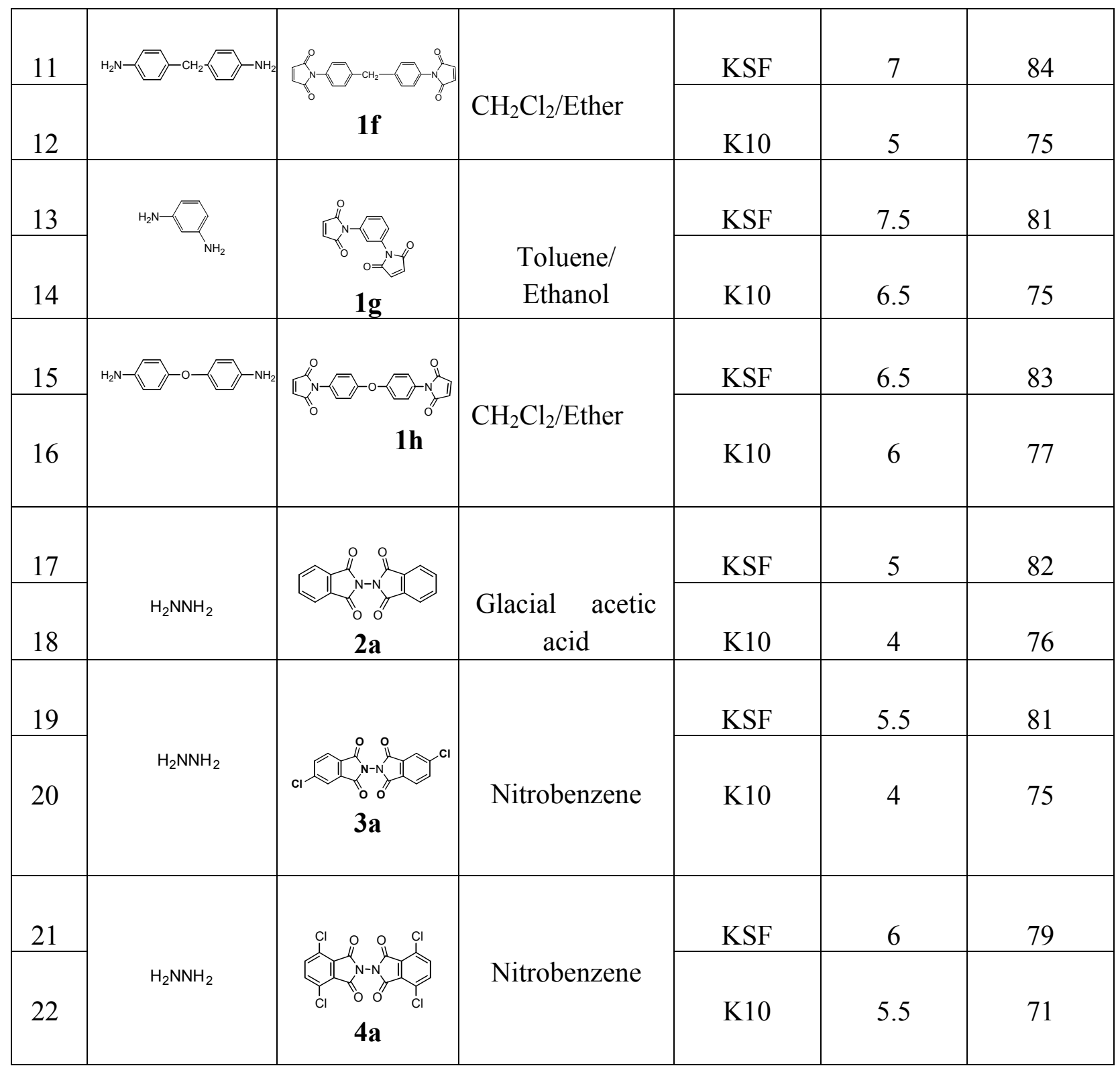

* All of the isolated products are well known and their spectra and physical data have been reported in the literatures.

** Isolated yields after recrystallization.

\section{Experimental Section}

General procedures. Melting points were measured on an Electrothermal 9100 apparatus. Elemental analyses were performed using a Heraeus CHN-O-Rapid analyzer. Reactions were conducted in a commercial microwave oven model Delonghi MW 545. Chemicals were 
purchased from the Fluka (montmorillonite KSF and montmorillonite K-10 clays) and Aldrich (anhydrides, diamines and solvents) chemical companies. The relevant products were characterized by the comparison of their spectra (IR, ${ }^{1} \mathrm{H}$ NMR and ${ }^{13} \mathrm{C} N \mathrm{NM}$ ), elemental analysis and physical data with the authentic samples. $\mathrm{H}$ NMR and $\mathrm{C}$ NMR spectra were recorded on a FT-NMR JEOL FX 90Q spectrometer. Chemical shifts were measured in ppm from TMS. DMSO- $\mathrm{d}_{6}$ was used as a solvent as well as the internal standard. The IR spectra were obtained on a Perkin Elmer FT IR GX instrument in $\mathrm{KBr}$ discs.

$\boldsymbol{N}$ - Phthalimidophthalimide (2a). In a typical experiment, phthalic anhydride (2.2 mmole, 0.33 g) and montmorillonite KSF clay (1g) mixed, grinded properly, placed in a clean and dry beaker and hydrazine hydrate ( 1 mmole, $0.05 \mathrm{~g}$ ) was then added. The reaction mixture preheated in a microwave oven for $1 \mathrm{~min}$ (power $600 \mathrm{~W}$ ) and the heating continued for 4 min (power $780 \mathrm{~W}$ ) to complete the reaction which was monitored by TLC using n-hexane/acetone as an eluent (3:7). The reaction mixture were allowed to cool to the room temperature and the resulting product extracted into $\mathrm{CH}_{2} \mathrm{Cl}_{2}(2 \times 20 \mathrm{~mL})$. The montmorillonite $\mathrm{KSF}$ clay was filtered off and the solvent removed by rotary. Solid clay portion was washed with methanol and dried at $120^{\circ} \mathrm{C}$ under the reduced pressure for $5 \mathrm{~h}$ to be reused in the subsequent reactions. The obtained product was washed with distilled and warm water, dried in the oven $\left(50^{\circ} \mathrm{C}, 4 \mathrm{~h}\right)$ and recrystallized from acetic acid to collect $N$-phthalimidophthalimide (2a) as colorless prisms $(9.66 \mathrm{~g}, 82 \%), \mathrm{mp}>300^{\circ} \mathrm{C}$ (lit. ${ }^{22 \mathrm{e}} 44.5 \%$, mp $311-313^{\circ} \mathrm{C}$, reaction time $1 \mathrm{~h}$ in refluxing with acetic acid). IR $(\mathrm{KBr}) \mathrm{cm}^{-1}$ : 3028, 1783, 1745, 1657, 1601, 1493, 1377, 683. H NMR (DMSO-d 6 ): $\delta 7.71$ (4H, dd, Ar), 8.15 (4H, d, Ar), ${ }^{13} \mathrm{C}$ NMR (DMSO-d 6 ): $\delta 127.4,132.0,132.3$, 165.5. Anal. Calcd. for $\mathrm{C}_{16} \mathrm{H}_{8} \mathrm{~N}_{2} \mathrm{O}_{4}: \mathrm{C}$, 65.75; H, 2.80; N, 9.60. Found: C, 65.14; H, 2.23; N, 9.31\%.

Bismaleimide (1a). Yield $86 \%$, mp $256-258^{\circ} \mathrm{C}\left(1 \mathrm{it} .{ }^{22 \mathrm{a}} 259-260^{\circ} \mathrm{C}\right) . \mathrm{IR}(\mathrm{KBr}) \mathrm{cm}^{-1}: 3026,2938$, 2918, 1780, 1714, 1612, 1385, 709. H NMR (DMSO-d 6 ): $\delta 6.95$ (4H, s, Vinilic), ${ }^{13} \mathrm{C}$ NMR (DMSO-d $\mathrm{d}_{6}$ ): $\delta 136.4,162.9$. Anal. Calcd. for $\mathrm{C}_{8} \mathrm{H}_{4} \mathrm{~N}_{2} \mathrm{O}_{4}: \mathrm{C}, 50.0 ; \mathrm{H}, 2.08 ; \mathrm{N}, 14.58$. Found: C, 49.37; H, 2.43; N, 14.06\%.

1,1'-Ethylenebismaleimide (1b). Yield 74\%, mp 187-190 ${ }^{\circ} \mathrm{C}$ (lit. $\left.{ }^{22 \mathrm{~b}} 190-192^{\circ} \mathrm{C}\right) . \mathrm{IR}(\mathrm{KBr}) \mathrm{cm}^{-1}$ : 2926, 1774, 1709, 1619, 1432, 719. H NMR (DMSO-d $)_{6}: \delta 3.74\left(4 \mathrm{H}, \mathrm{t}, 2 \mathrm{CH}_{2}\right), 6.92(4 \mathrm{H}, \mathrm{s}$, Vinilic), ${ }^{13} \mathrm{C}$ NMR (DMSO-d 6 ): $\delta 39.4,136.6,164.5$. Anal. Calcd. for $\mathrm{C}_{10} \mathrm{H}_{8} \mathrm{~N}_{2} \mathrm{O}_{4}: \mathrm{C}, 54.55 ; \mathrm{H}$, 3.66; N, 12.72. Found: C, 54.81; H, 3.97; N, 12.47\%.

1,1'-Hexamethylenebismaleimide (1c). Yield $68 \%$, mp $136-138^{\circ} \mathrm{C}$ (lit. $\left.{ }^{22 \mathrm{~b}} 139-140^{\circ} \mathrm{C}\right)$. IR (KBr) $\mathrm{cm}^{-1}: 2914,1768,1712,1623,1468,1378,695$. H NMR (DMSO-d 6 ): $\delta 1.39\left(2 \mathrm{H}, \mathrm{t}, 3-\mathrm{CH}_{2}\right), 1.55$ $\left(2 \mathrm{H}, \mathrm{m}, 2-\mathrm{CH}_{2}\right), 3.48\left(2 \mathrm{H}, \mathrm{m}, 1-\mathrm{CH}_{2}\right),{ }^{13} \mathrm{C} \mathrm{NMR}\left(\mathrm{DMSO}-\mathrm{d}_{6}\right): \delta 24.5,28.7,40.8,136.5,164.6$. Anal. Calcd. for $\mathrm{C}_{14} \mathrm{H}_{16} \mathrm{~N}_{2} \mathrm{O}_{4}$ : C, 60.86; H, 5.84; N, 10.14. Found: C, 60.07; H, 5.36; N, 9.55\%.

1,1'-(1,4-Phenylene)bismaleimide (1d). Yield $89 \%, \mathrm{mp}>300^{\circ} \mathrm{C}$ (lit. ${ }_{1}^{22 \mathrm{~b}} 346-350{ }^{\circ} \mathrm{C}$ ). IR (KBr) $\mathrm{cm}^{-1}: 3461,3435,3169,3107,1778,1717,1612,1602,1457,1398,699$. H NMR (DMSO-d $): \delta$ 7.2 (4H, s, Vinilic), 7.62-7.40 (4H, d, $\left.\mathrm{C}_{6} \mathrm{H}_{4}\right),{ }^{13} \mathrm{C}$ NMR (DMSO- $\left.\mathrm{d}_{6}\right): \delta 119.8,132.5,136.0,163.3$. Anal. Calcd. for $\mathrm{C}_{14} \mathrm{H}_{8} \mathrm{~N}_{2} \mathrm{O}_{4}$ : C, 62.69; H, 3.01; N, 10.45. Found: C, 63.18; H, 3.49; N, 10.12\%. 
1,1'-(Di-1,4-phenylene)bismaleimide (1e). Yield $87 \%, \mathrm{mp}>300^{\circ} \mathrm{C}\left(1 \mathrm{it} .{ }^{22 \mathrm{~b}} 340-349^{\circ} \mathrm{C}\right) . \mathrm{IR}(\mathrm{KBr})$ $\mathrm{cm}^{-1}: 3467,3429,3153,3108,1765,1711,1619,1457,1385,691$. H NMR (DMSO-d 6 ): $\delta 7.16$ (4H, s, Vinilic), 7.46-7.70 (8H, m, $\left.2 \mathrm{C}_{6} \mathrm{H}_{4}\right),{ }^{13} \mathrm{C}$ NMR (DMSO- $\left.\mathrm{d}_{6}\right): \delta 120.8,127.6,132.4,136.0$, 137.3, 161.8. Anal. Calcd. for $\mathrm{C}_{20} \mathrm{H}_{12} \mathrm{~N}_{2} \mathrm{O}_{4}$ : C, 69.73; H, 3.51; N, 8.14. Found: C, 69.27; H, 3.75; $\mathrm{N}, 8.73 \%$.

1,1'-(Methylenedi-1,4-phenylene)bismaleimide (1f). Yield $84 \%$, mp $158-161^{\circ} \mathrm{C}$ (lit. $^{22 \mathrm{c}} 161$ $\left.162^{\circ} \mathrm{C}\right)$. IR (KBr) cm ${ }^{-1}: 3079,3036,1707,1521,1392,1365,1153,886 .{ }^{1} \mathrm{H}$ NMR (DMSO-d $\left.{ }_{6}\right): \delta$ $4.02\left(2 \mathrm{H}, \mathrm{s}, \mathrm{CH}_{2}\right), 6.98$ (4H, s, Vinilic), 7.28-7.38 (8H, m, $\left.2 \mathrm{C}_{6} \mathrm{H}_{4}\right),{ }^{13} \mathrm{C}$ NMR (DMSO-d 6 ): $\delta$ 40.83, 126.15, 129.11, 129.64, 133.09, 139.27, 169.43. Anal. Calcd. for $\mathrm{C}_{21} \mathrm{H}_{14} \mathrm{~N}_{2} \mathrm{O}_{4}$ : C, 70.39; H, 3.94; N, 7.82. Found: C, 70.79; H, 4.27; N, 7.58\%.

1,1'-(1,3-Phenylene)bismaleimide (1g). Yield 81\%, mp 204-206 ${ }^{\circ} \mathrm{C}$ (lit. $\left.{ }^{22 \mathrm{~d}}>200^{\circ} \mathrm{C}\right)$. IR (KBr) $\mathrm{cm}^{-1}: 3451,3163,3037,1708,1615,1502,1427,1377,1131,682$. H NMR (DMSO-d $): \delta 7.1$ (4H, s, Vinilic), 7.22-7.49 (4H, m, $\left.\mathrm{C}_{6} \mathrm{H}_{4}\right),{ }^{13} \mathrm{C}$ NMR (DMSO-d 6 ): $\delta 121.78,124.5,128.95$, 132.06, 135.4, 168.8. Anal. Calcd. for $\mathrm{C}_{14} \mathrm{H}_{8} \mathrm{~N}_{2} \mathrm{O}_{4}$ : C, 62.69; H, 3.01; N, 10.45. Found: C, 61.96; H, 3.32; N, 9.71\%.

1,1'-(Oxydi-1,4-phenylene)bismaleimide (1h). Yield 83\%, mp 182-186 ${ }^{\circ} \mathrm{C}$ (lit. $\left.{ }^{22 \mathrm{c}} 185-186^{\circ} \mathrm{C}\right)$. IR $(\mathrm{KBr}) \mathrm{cm}^{-1}: 3082,1780,1718,1603,1497,1342,1258,1193,873 .{ }^{1} \mathrm{H}$ NMR (DMSO-d 6$): \delta$ 7.02 (4H, s, Vinilic), 7.54-7.82 (8H, m, $\left.2 \mathrm{C}_{6} \mathrm{H}_{4}\right),{ }^{13} \mathrm{C}$ NMR (DMSO-d $\left.{ }_{6}\right): \delta 56.7,114.1,128.6$, 129.3, 136.7, 164.9. Anal. Calcd. for $\mathrm{C}_{20} \mathrm{H}_{12} \mathrm{~N}_{2} \mathrm{O}_{5}$ : C, 66.66; H, 3.33; N, 7.77. Found: C, 65.94; H, 3.76; N, 7.18\%.

4,4'-Dichloro- $\boldsymbol{N}$-phthalimidophthalimide (3a). Yield $81 \%, \underset{1}{\operatorname{mp}}>300^{\circ} \mathrm{C}$ (lit. ${ }^{22 \mathrm{f}} 303-305^{\circ} \mathrm{C}$ ). IR $(\mathrm{KBr}) \mathrm{cm}^{-1}: 3092,2957,2862,1774,1719,1609,1398,690$. H NMR (DMSO-d 6 ): $\delta$ 7.70-8.14 $(6 \mathrm{H}, \mathrm{m}, \mathrm{Ar}) .{ }^{13} \mathrm{C} \mathrm{NMR}\left(\mathrm{DMSO}-\mathrm{d}_{6}\right): \delta 165.3,137.3,133.8,132.4,130.4,128.6,127.9$. Anal. Calcd. for $\mathrm{C}_{16} \mathrm{H}_{6} \mathrm{~N}_{2} \mathrm{O}_{4} \mathrm{Cl}_{2}$ : C, 53.18; $\mathrm{H}, 1.66 ; \mathrm{N}, 7.75$. Found: $\mathrm{C}, 52.85 ; \mathrm{H}, 1.87 ; \mathrm{N}, 7.02 \%$.

3,6,3',6'-Tetrachloro- $N$-phthalimidophthalimide (4a). Yield $79 \%, \mathrm{mp}>300^{\circ} \mathrm{C}\left(\right.$ lit. $^{22 \mathrm{e}}>350^{\circ} \mathrm{C}$ ). IR (KBr) $\mathrm{cm}^{-1}: 3097,2969,2878,1790,1727,1596,1394,716$. H NMR (DMSO-d 6 ): $\delta 7.64$ (4H, s, Ar). ${ }^{13} \mathrm{C}$ NMR (DMSO-d 6 ): $\delta 165.8,134.1,133.7,130.8$. Anal. Calcd. for $\mathrm{C}_{16} \mathrm{H}_{4} \mathrm{~N}_{2} \mathrm{O}_{4} \mathrm{Cl}_{2}$ : C, 44.6; H, 0.9; N, 6.5. Found: C, 44.81; H, 1.17; N, 8.83\%.

\section{Conclusions}

In summary, the paper describes a simple and efficient method for the synthesis of bismaleimides and bisphthalimides from the relevant anhydrides on montmorillonite KSF and montmorillonite K-10 clays as heterogeneous catalysts by the help of microwave irradiation. The notable features of this procedure are the solvent-free reaction conditions, high yields of products, cleaner reaction profiles and availability of the reagents at low cost and enhanced rates which makes it a useful and attractive process for the synthesis. The simple experimental and 
product isolation procedures as well as the easy recovery and reuse of the natural clays are expected to play an important role in development of the new method.

\section{Acknowledgements}

The authors gratefully acknowledge the financial supports from the Bu Ali Sina University, Hamedan, 6517838683, Iran.

\section{References}

1. (a) Da Settimo, A.; Primofiore, G.; Da Settimo, F.; Simorini, F.; La Motta, C.; Martinelli A.; Boldrini, E. Eur. J. Med. Chem. 1996, 31, 49. (b) Langmuir, M.; Yang, E. J. R.; Moussa, A. M.; Laura R.; Lecompte, K. A. Tetrahedron Lett. 1995, 36, 3989. (c) Mayer A.; Neuenhofer, S. Angew. Chem. Int. Ed. Engl. 1994, 33, 1044.

2. (a) Rusiecki, V. K.; Warne, S. A. Biorg. Med. Chem. Lett. 1993, 3, 707. (b) Wong, S. S. Chemistry of Protein Conjugation and Cross-linking, CRC Press: Boca Raton, 1991. (c) Janda, D. K.; Ashley, A. J.; Jones, T. M.; McLeod, D. A.; Schloeder, D. M.; Weinhouse, M. I. J. Am. Chem. Soc. 1990, 112, 8886 .

3. (a) FitzGerald, D.; Idziorek, T.; Batra, J. K.; Willingham M.; Pastan, I. Bioconjugate Chem. 1990, 1, 264. (b) Maeda, M.; Kumano, A.; Tirrell, D. A. J. Am. Chem. Soc. 1988, 110, 7455.

4. (a) Ohkubo, M.; Nishimura, T.; Jona, H.; Honma, T.; Morishima, H. Tetrahedron 1996, 52, 8099. (b) Hamper, B. C.; Dukesherer D. R.; South, M. S. Tetrahedron Lett. 1996, 37, 3671. (c) Yoon, U. C.; Kim, D. K.; Lee, C. W.; Choi, Y. S.; Lee,Y. J.; Ammon, H. L.; Mariano, P. S. J. Am. Chem. Soc. 1995, 117, 2698.

5. (a) Kang, J.; Lee, J. W.; Kim, J. J.; Pyun, C. Tetrahedron Lett., 1995, 36, 4265. (b) Faul, M. M.; Sullivan, K. A.; Winneroski, L. L. Synthesis 1995, 1511. (c) Romagnoli, R.; Roos, E. C.; Hiemstra, H.; Moolenaar, M. J.; Speckamp, W. N.; Kaptein, B.; Schoemaker, H. E. Tetrahedron Lett., 1994, 35, 1087.

6. (a) Arai, Y.; Matsui, M.; Fujii, A.; Kontani, T.; Ohno, T.; Koizumi, T.; Shiro, M. J. Chem. Soc., Perkin Trans.1 1994, 25. (b) Baldwin, S. W.; Greenspan, P.; Alaimo, C.; McPhail, A. T. Tetrahedron Lett. 1991, 32, 5877. (c) Miller, S. A.; Chamberlin, A. R. J. Org. Chem. 1989, 54, 2502.

7. (a) Iijima, T.; Suzuki, N.; Fukuda, W.; Tomoi, M. J. Eur. Polym. 1995, 31, 775. (b) Amou, S.; Nishimura, S.; Takahashi, A.; Hagiwara, T.; Hamana H.; Narita, T. J. Polym. Sci. Tech. 1994, 51, 764.

8. (a) Bharel, R.; Choudhary V.; Varma, I. K. J. Appl. Polym. Sci. 1993, 49, 31. (b) Dean, B. D. J. Appl. Polym. Sci. 1987, 33, 2259. 
9. (a) Gorin, G.; Martic P. A.; Doughty, G. Arch. Biochem. Biophys. 1966, 115, 593. (b) Corrie, J. E. T. J. Chem. Soc., Perkin Trans. 1 1994, 2975. (c) Kunicki, T. J.; Nugent, D. J.; Piotrowicz, R. S.; Lai, C. S. Biochemistry 1986, 25, 4979.

10. (a) Wong, S. S. Chemistry of Protein Conjugation and Crosslinking, CRC Press: Boca Raton, 1991. (b) Smyth, G. E.; Colman, R. F. J. Biol. Chem., 1991, 266, 14918.

11. (a) Janda, K. D.; Ashley, J. A. ; Jones, T. M.; McLeod, D. A.; Chloeder S. D. M.; Weinhouse, M. I. J. Am. Chem. Soc. 1990, 112, 8886. (b) Pai L. H.; Pastan, I. JAMA. 1993, 269, 78. (c) Rusiecki V. K.; Warne, S. A. Biorg. Med. Chem. Lett. 1993, 3, 707.

12. (a) Baldwin, S. W.; Greenspan, P.; Alaimo C.; McPhail, A. T. Tetrahedron Lett. 1991, 32, 5877. (b) Arai, Y.; Matsui, M.; Fujii, A.; Kontani, T.; Ohno, T.; Koizumi T.; Shiro, M. J. Chem. Soc., Perkin Trans. 1 1994, 25.

13. (a) Grigg, R.; Surendrakumar, S.; Thianpatanagul S.; Vipond, D. J. Chem. Soc. Perkin Trans. 1 1988, 2693. (b) Preston, P. N.; Shah, V. K.; Simpson, S. W.; Souter I.; Stewart, N. J. Macromolecules 1994, 27, 1147.

14. (a) Walker, M. A. J. Org. Chem. 1995, 60, 5352. (b) Walker, M. A. Tetrahedron Lett. 1994, 35, 665. (c) Corrie, J. E. T. J. Chem. Soc., Perkin Trans. 1 1994, 2975. (d) Gill, G. B.; James, G. D.; Oates, K. V.; Pattenden, G. J. Chem. Soc., Perkin Trans. 1 1993, 2567.

15. (a) Braish, T. F.; Fox, D. E. Synlett 1992, 979. (b) Dorta, R. L.; Francisco, C. G.; Suarez, E. Tetrahedron Lett. 1991, 35, 1083. (c) Nielsen, O.; Buchardt, O. Synthesis 1991, 819.

16. (a) Rangnekar, V. M.; Bharmaria, R. P.; Khadse, B. G. Ind. J. Chem. 1986, 25B, 342. (b) Schwartz, A. L.; Lerner L. M. J. Org. Chem. 1974, 39, 21. (c) Mehta, N. B.; Phillips, A. P.; Lui, F. F.; Brooks, R. E. J. Org. Chem. 1960, 25, 1012.

17. Krstic, L. J.; Sukdolak, S.; Solujic, S. J. Serb. Chem. Soc. 2002, 67 (5), 325.

18. (a) Caddick, S. Tetrahedron 1995, 51, 10403. (b) Lidstrom, P.; Tierney, J.; Wathey B.; Westman, J. Tetrahedron 2001, 57, 9225. (c) Lauren, R.; Leporterie, A.; Dubac, J.; Berlan, J.; Lauverie S.; Audhuy, F. M. J. Org. Chem. 1992, 57, 7099.

19. (a) Bose, A. K.; Manhas, M. S.; Ganguly, S. N.; Sharma, A. H.; Banik, B. K. Synthesis 2002, 1578. (b) Loupy, A.; Petit, A.; Hamelin, J.; Texier-Boullet, F.; Jacquault, P.; Mathe, D. Synthesis 1998, 1213. (c) Varma, R. S. Green Chem. 1999, 1, 43. (d) Varma, R. S. Clean Prod. Pros. 1999, 1, 132.

20. (a) Bram, G.; Loupy, A.; Villemerin, D. Solid support and Catalysed in Organic Chemistry, Ellis Horwood, London, 1992. (b) Boruah, A.; Baruah, M.; Prajapti, D.; Sandhu, J. S. Chem. Lett. 1996, 965.

21. (a) Habibi, D; Marvi, O. J. Serb. Chem. Soc. 2005, 70(4), 579. (b) Habibi, D; Marvi, O. Catal. Commun., accepted, in press.

22. (a) Feuer, H.; Rubinstein, H. J. Am. Chem. Soc. 1958, 80, 5873. (b) Kovacic, P.; Hein, R. W. J. Am. Chem. Soc. 1959, 81, 1187. (c) Sun, K. K. Macromolecules 1987, 20, 726. (d) Lee, I. J.; Choi, H. W.; Nho, Y. C.; Suh, D. H. J. Appl. Polym. Sci. 2003, 88, 2339. (e) Drew, H. D. K.; Hatt, H. H.; J. Chem. Soc. 1937, 16, 16. (f) Hay, A. S.; Ghasemi, H. US Patent 5,359,092 (Oct. 25, 1994). (g) Woods, H. J. US Patent 4,042,633 (Aug. 16, 1977). 